\title{
Article
}

\section{Electronic Structure Tunability by Periodic Meta-Ligand Spacing in One-Dimensional Organic Semiconductors}

Ignacio Piquero-Zulaica, Aran Garcia-Lekue, Luciano Colazzo, Claudio K.

Krug, Mohammed S. G. Mohammed, Zakaria M. Abd El-Fattah, J. Michael

Gottfried, Dimas G. de Oteyza, J. Enrique Ortega, and Jorge Lobo-Checa

ACS Nano, Just Accepted Manuscript • DOI: 10.1021/acsnano.8b06536 • Publication Date (Web): 08 Oct 2018

Downloaded from http://pubs.acs.org on October 8, 2018

\section{Just Accepted}

"Just Accepted" manuscripts have been peer-reviewed and accepted for publication. They are posted online prior to technical editing, formatting for publication and author proofing. The American Chemical Society provides "Just Accepted" as a service to the research community to expedite the dissemination of scientific material as soon as possible after acceptance. "Just Accepted" manuscripts appear in full in PDF format accompanied by an HTML abstract. "Just Accepted" manuscripts have been fully peer reviewed, but should not be considered the official version of record. They are citable by the Digital Object Identifier (DOI®). "Just Accepted" is an optional service offered to authors. Therefore, the "Just Accepted" Web site may not include all articles that will be published in the journal. After a manuscript is technically edited and formatted, it will be removed from the "Just Accepted" Web site and published as an ASAP article. Note that technical editing may introduce minor changes to the manuscript text and/or graphics which could affect content, and all legal disclaimers and ethical guidelines that apply to the journal pertain. ACS cannot be held responsible for errors or consequences arising from the use of information contained in these "Just Accepted" manuscripts. 


\title{
Electronic Structure Tunability by Periodic
}

\section{Meta-Ligand Spacing in One-Dimensional \\ Organic Semiconductors}

\author{
Ignacio Piquero-Zulaica, ${ }^{*} \dagger$ Aran Garcia-Lekue, ${ }^{\ddagger}, \operatorname{Tuciano}^{\dagger}$ Colazzo, ${ }^{\ddagger}$ Claudio K. \\ Krug, ${ }^{\S}$ Mohammed S. G. Mohammed, ${ }^{\ddagger} \dagger$ Zakaria M. Abd El-Fattah,,$\|, \perp$ J. Michael \\ Gottfried, Dimas G. de Oteyza, ${ }^{\dagger, \ddagger}$, J. Enrique Ortega, ${ }^{\dagger, \ddagger}, \#$ and Jorge \\ Lobo-Checa*,@, $\triangle$
}

$\dagger$ Centro de Física de Materiales CSIC/UPV-EHU-Materials Physics Center, Paseo Manuel de Lardizabal 5, E-20018 San Sebastián, Spain

$\ddagger$ Donostia International Physics Center (DIPC), Paseo Manuel de Lardizabal 4, E-20018 Donostia-San Sebastián, Spain

IIkerbasque, Basque Foundation for Science, 48011 Bilbao, Spain

$\S$ Fachbereich Chemie, Philipps-Universität Marburg, Hans-Meerwein-Str. 4, 35032 Marburg, Germany

||ICFO-Institut de Ciencies Fotoniques, The Barcelona Institute of Science and Technology, 08860 Castelldefels, Barcelona, Spain

$\perp$ Physics Department, Faculty of Science, Al-Azhar University, Nasr City, E-11884 Cairo, Egypt

\#Dpto. Fisica Aplicada I, Universidad del País Vasco, E-20018 San Sebastián, Spain @Instituto de Ciencia de Materiales de Aragón (ICMA), CSIC-Universidad de Zaragoza, E-50009 Zaragoza, Spain

$\triangle$ Departamento de Física de la Materia Condensada, Universidad de Zaragoza, E-50009 Zaragoza, Spain

E-mail: ipiquerozulaica@gmail.com; jorge.lobo@csic.es

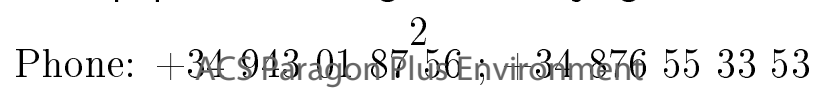




\begin{abstract}
Designing molecular organic semiconductors with distinct frontier orbitals is key for the development of devices with desirable properties. Generating defined organic nanostructures with atomic precision can be accomplished by on-surface synthesis. We use this 'dry' chemistry to introduce topological variations in a conjugated poly-(paraphenylene) chain in the form of meta-junctions. As evidenced by STM and LEED, we produce a macroscopically ordered, monolayer thin zigzag chain film on a vicinal silver crystal. These cross-conjugated nanostructures are expected to display altered electronic properties, which are now unravelled by highly complementary experimental techniques (ARPES and STS) and theoretical calculations (DFT and EPWE). We find that meta-junctions dominate the weakly dispersive band structure, while the bandgap is tunable by altering the linear segment's length. These periodic topology effects induce significant loss of the electronic coupling between neighboring linear segments leading to partial electron confinement in the form of weakly coupled Quantum Dots. Such periodic quantum interference effects determine the overall semiconducting character and functionality of the chains.
\end{abstract}

\title{
Keywords
}

On-surface synthesis, cross-conjugated polymers, ultra-thin organic films, vicinal surfaces, electronic structure, electron confinement.

Conjugated polymers in the form of molecular chains are extensively used in industry as light emitting materials, photocatalysts, solar cells and biosensors due to their large and tunable bandgaps. ${ }^{1-4}$ Control over their electronic properties is accomplished through topological functionalization of these $\pi$-conjugated oligophenylene chains, i.e. modification of their conductive pathways. In particular, changes of conjugation (from linear to crossconjugation) by precise transitions from para- to meta-ligand substitutions ${ }^{5,6}$ weaken the

electronic communication between the repeating units of the polymer. ${ }^{7}$ Such modifications 
have also been described as quantum interference electron pathways, ${ }^{8-10}$ and bear predicted effects such as scarcely dispersive bands, ${ }^{5}$ wider bandgap,${ }^{11}$ distinct optical properties, ${ }^{4}$ electronic switching capabilites ${ }^{12}$ and low conductance properties. ${ }^{8-10,13,14}$ However, periodic meta-junction zigzag chains may also show enhanced charge mobility as compared to their poly-(para-phenylene) counterparts, reaching values comparable to those of amorphous silicon. $^{15}$

Despite this wealth of industrially attractive properties of cross-conjugated polymers, key fundamental information, such as the predicted electronic structure awaits experimental validation. Such deficiency of fundamental knowledge limits the confidence in the existing predictions, according to which topology is expected to affect the electronic properties of the polymer. Several obstacles are responsible for the lack of the aforementioned experimental confirmation: i) the need of generating atomically identical chains exhibiting repeated parato meta-ligand substituted units, ii) the synthesis of well-aligned chains, to be probed by non-local, averaging spectroscopies, iii) the minimization of lateral interactions, prone to affect their intrinsic band structure, and iv) the right choice of a support that sufficiently decouples the electronic signal from the investigated oligophenylene chains.

To overcome such obstacles, solutions can be found within the context of Surface Science. Particularly, the first prerequisite for obtaining perfectly reproducible cross-conjugated zigzag polymers can be accomplished by bottom-up on-surface synthesis. Surface-assisted C-C coupling processes have been recently applied to generate graphene nanoribbons (GNRs) with different edge terminations and widths, ${ }^{16-21}$ and other types of oligophenylene chains. ${ }^{22-25}$ Secondly, the chain alignment for non-local characterization can be achieved by the use of nanotemplated substrates, such as vicinal surfaces. ${ }^{26-29}$ These special surfaces have been successfully used for the macroscopic alignment of carbon-based chains, a fundamental requirement for angle resolved photoemission (ARPES) experiments. ${ }^{23,24,30,31}$ With respect to the minimization of lateral interchain coupling, this is an inherent feature of the Ullmanntype surface reactions ${ }^{22}$ since the halogens are cleaved during the synthesis positioning them- 
selves between neighboring chains. ${ }^{23,24,32}$ These adatoms are reported to laterally decouple adjoining chains, without affecting the polymer's band structure, except for a minimal rigid energy shift similar to doping effects. ${ }^{24,33}$ Finally, the substrate plays a fundamental role as a catalyst of the Ullmann reaction, making its choice crucial for a successful oligomer coupling. Good candidates that present excellent yields, control and reproducibility are the closed packed surfaces of coinage metals, which are extensively used for Ullmann-type surface reactions. Among these, silver stands out as a promising substrate, since it weakly interacts with the products while exhibiting large adsorbate diffusion rates. ${ }^{34}$ Moreover, its $d$-bands are furthest from the Fermi level (below $-3 \mathrm{eV}$ ), allowing a wide energy range for the study of the chain's band structure (see Figure S1 in S.I.).

In this work, we have overcome all the aforementioned obstacles and have generated an extended film of atomically precise zigzag chains on a vicinal $\operatorname{Ag}(111)$ surface, as evidenced by scanning tunneling microscopy (STM) and low energy electron diffraction (LEED). The electronic band structure of such films has been unravelled by means of ARPES and complemented by scanning tunneling spectroscopy (STS) measurements on $\operatorname{Ag}(111)$. In this way, we determine the experimental energy gap and visualize the spatial distribution of the frontier orbitals. Such wealth of experimental information has been clarified and expanded by a comprehensive set of density functional theory (DFT) calculations and electron plane wave expansion (EPWE) simulations.

\section{Results and Discussion}

We have produced a monolayer film of cross-conjugated zigzag chains from the surface polymerization of the 4,4"-dibromo-meta terphenyl (DMTP) molecular aromatic precursors via C-C coupling (see Figure 1a and Methods section). The template of choice is a vicinal $\operatorname{Ag}(111)$ crystal surface with linear, monoatomic steps running parallel to the [11-2] direc- 
tion $^{29}$ that corresponds to the so-called fully-kinked (100\% kinked) configuration of the stepedge. We used this particular substrate since it provides a higher flexibility to reconstruct and therefore accommodate the produced zigzag structures more efficiently (cf. Methods section and Figure S1 in S.I.). Indeed, we can already disclose that we achieved an excellent film featuring a high yield of well-ordered and aligned zigzag chains.

The formed zigzag chains appear practically planar on the surface (Figure 1b) and are covalently bonded displaying the characteristic phenyl-phenyl distance of $a \sim 4.3 \AA$ along the straight segments and a superperiodicity of $L \sim 2.24 \mathrm{~nm}$ between equivalent elbows. ${ }^{22,35}$ The unit cell of the chain features two straight subunits made up of two phenyl rings (in para-positions) linked to two edge rings acting as meta-junctions (Figure 1a). Note that in the STM image these chains are separated by spherical features attributed to Br atoms split off from the precursor molecules at the initial step of the on-surface reaction. ${ }^{36-43}$ The LEED pattern reveals that the organic chains are aligned parallel to the steps and show long-range order as they are commensurate with the underlying substrate (Figure 1b and Figure S2 in S.I.). Particularly, the main silver diffraction spots (red and green circles) are surrounded by a set of spots aligned along the average step direction yielding a $(9,5 ; 0,4)$ superstructure.

Our STM and LEED structural results contain the required ingredients (atomic precision of the structure, defined alignment, long-range order and minimization of lateral interactions by $\mathrm{Br}$ adatom presence) to expect the existence of a defined and coherent electronic band structure from these chains. Figures 1c-e show the second derivative (to enhance the details) of the ARPES spectral weight obtained from such a film saturating the surface (raw data is shown in Figure S3 of the S. I.). The resulting electronic structure in the direction parallel to the average step direction and the main axis of the zigzag chains ( $E$ vs $k_{y}$ with $k_{x}=0.1 \AA^{-1}$ ), exhibits weakly dispersive bands between $-1.8 \mathrm{eV}$ and $-3.5 \mathrm{eV}$, separated by $\mathrm{a} \sim 0.6 \mathrm{eV}$ gap (cf. Figure 1c). None of these ARPES features are observable on the pristine substrate (cf. Figure S1 in S.I.). A closer inspection reveals that each one of them consists 
a

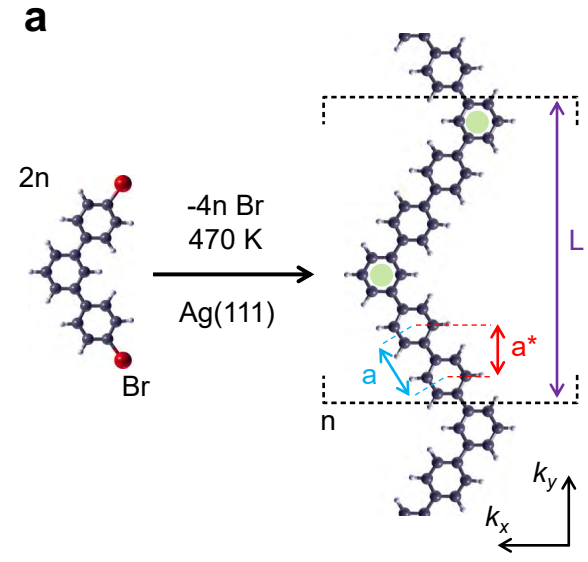

C

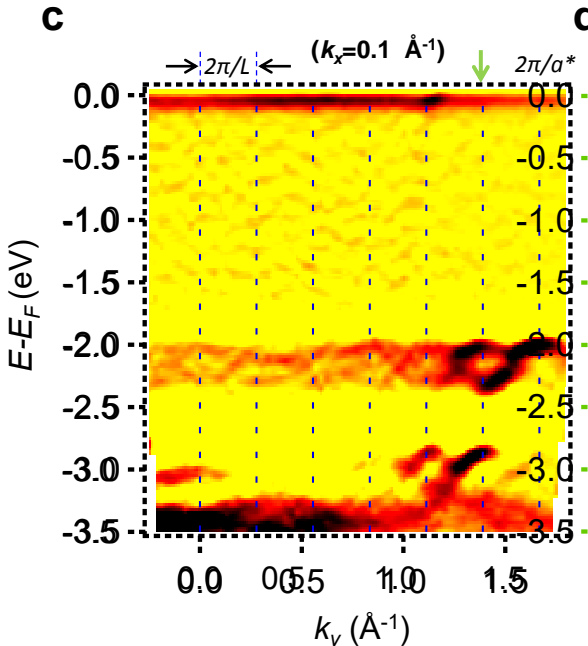

b

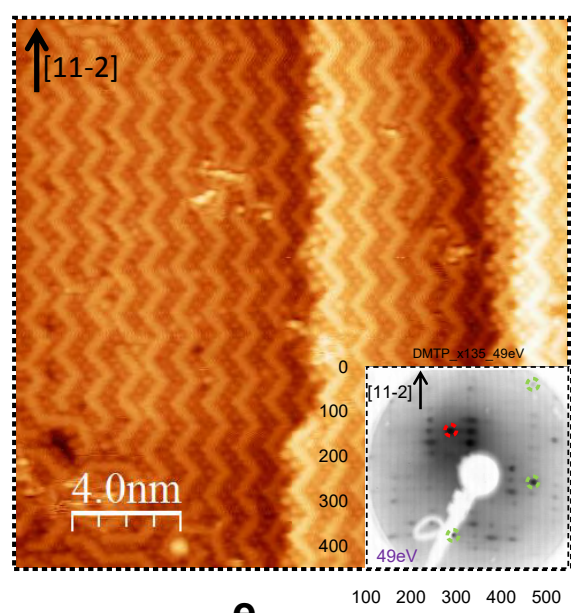

$\left(k_{y}=1.39 \AA^{-1}\right)$

e

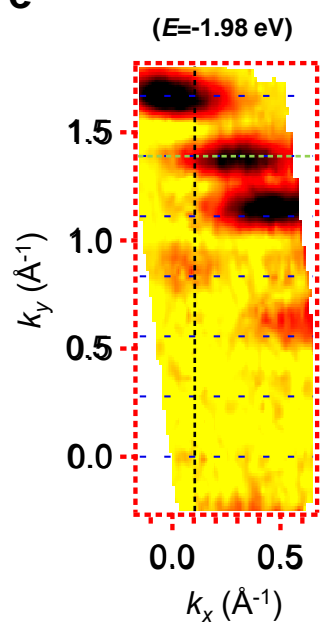

Figure 1: Structural arrangement and ARPES electronic band structure of the zigzag chain film grown on a vicinal $\mathrm{Ag}(111)$ surface. (a) Schematic representation of the DMTP precursor and the resulting zigzag covalent chain, showing its characteristic lenghts: phenylphenyl distance $(a)$ and its projection along the chain's average direction $\left(a^{*}\right)$, and polymer superperiodicity $(L)$. (b) High resolution STM image after chain synthesis on a vicinal plane $\sim 3.6^{\circ}$ off from the (111) crystal position. The zigzag chains are separated by $\mathrm{Br}$ atoms and preferentially follow the step parallel direction ([11-2]). (STM parameters: $V=-394 \mathrm{mV}, I=234 \mathrm{pA}, T_{\text {sample }}=100 \mathrm{~K}$ ). Inset shows the LEED pattern after chain formation that exhibits single-domain, well-aligned arrangement. The superstructure spots are in registry with the circled main spots (in red the $(0,0)$ and in green the substrate's first order diffractions), implying commensurability with the terrace atoms (LEED parameters: $E_{k i n}=49 \mathrm{eV}, T_{\text {sample }}=300 \mathrm{~K}$ ). (c) ARPES experimental band structure of meta-junctioned cross-conjugated zigzag chains parallel to the average direction of chains and steps ( $E$ vs $k_{y}$ with $\left.k_{x}=0.1 \AA^{-1}\right)$. (d) Experimental band structure perpendicular to the chain average axis ( $E$ vs $k_{x}$, with $k_{y}=1.39 \AA^{-1}$ indicated by green arrow in (c)). (e) Isoenergetic cut $\left(k_{x}\right.$ vs $\left.k_{y}\right)$ at the top of the valence molecular band $(E=-1.98 \mathrm{eV}$, marked by red arrow in $(\mathrm{d}))$. The second derivative of the intensity is shown in a linear color scale (highest being black). (ARPES parameters: $h \nu=21.2 \mathrm{eV}, T_{\text {sample }}=150 \mathrm{~K}$ ). 
of a pair of anti-phase oscillatory bands (Figs. S3 and S4 in S.I.). The spectral intensity peaks around $2 \pi / a^{*}$, where $a^{*}$ represents the projected phenyl-phenyl distance along the average chain direction (Figure 1a), assuring its molecular origin. ${ }^{44}$ The faint replicas with $2 \pi / L$ periodicity (vertical dashed blue lines), stem from the zigzag chain superperiodicity $L$ (Figure 1a), in agreement with the STM dataset.

The 1D nature of these zigzag chains is demonstrated by the lack of dispersion perpendicular to the average chain axis. Figure $1 \mathrm{~d}$ shows a representative cut ( $E$ vs $k_{x}$ ) across the center of the $6^{\text {th }}$ Brillouin zone (green arrow at $k_{y}=1.39 \AA^{-1}$ in Figure 1c), where discrete flat bands can be observed. This confirms that they stem from different molecular orbitals of the zigzag polymer. ${ }^{45}$ The non-dispersive character at the top of the valence band (red arrow at $-1.98 \mathrm{eV})$ can also be traced from the isoenergetic cut $\left(k_{x}\right.$ vs $\left.k_{y}\right)$ shown in Figure 1e, where 1D polymer bands replicate at each Brillouin zone center, gaining intensity for the larger $k_{y}$ values. These photoemission intensity modulations have been simulated with the EPWE method, ${ }^{46,47}$ which confirms that these features are neither affected by the templating Ag surface nor by the presence of Br atoms intercalated between the chains (see Methods section and Figure S4 in S.I.). Indeed, we experimentally find that the presence of Br embedded in between the zigzag chains only causes a rigid shift of the molecular band structure by $200 \pm 50 \mathrm{meV}$ to higher energy, according to Figure S5 of the S.I. and in agreement with previous work $\cdot^{24,33}$

Our ARPES results suggest that the zigzag chains are largely decoupled from the metallic substrate since the observed molecular bands do not show signs of hybridization with the substrate in that energy window. Besides, the chains are semiconducting in nature with a bandgap certainly larger than $2 \mathrm{eV}$, since no other bands closer to the Fermi energy are observed in the occupied region. The band structure strongly contrasts with that of the poly-(para-phenylene) (called PPP hereafter) chains, which exhibits a single, highly dispersive molecular band across the entire Brillouin zone ${ }^{23-25}$ (Figure S6 in S.I.). Instead, it closely resembles the one predicted for poly-(meta-phenylene) (called PMP hereafter) chains, ${ }^{5}$ im- 
plying that the presence of meta-junctions strongly modifies the electronic structure of a polymeric chain ${ }^{13}$ (cf. Fig S7 of S.I.).

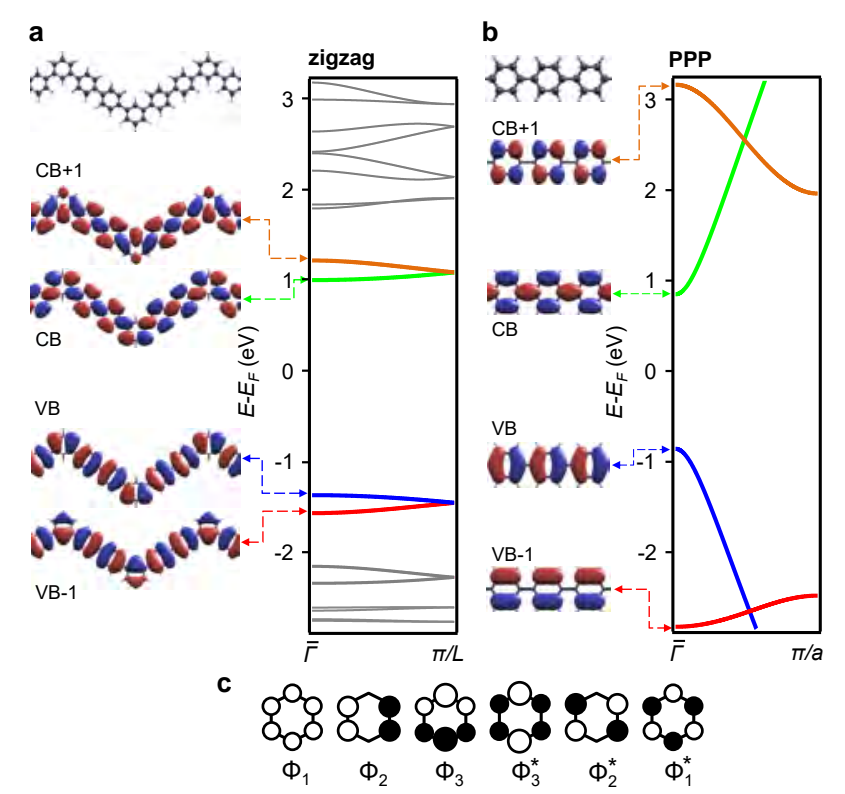

Figure 2: Comparison of molecular orbital shape and band structure between zigzag chains and straight PPP chains, as obtained from DFT calculations. The right plots in (a) and (b) show the calculated electronic band structure, where (a) corresponds to the zigzag polymer and (b) to PPP. The highly dispersive character of the PPP bands contrasts with the practically flat bands of the zigzag chains, accompanied by a notable difference in the frontier orbital bandgap. Left panels in (a) and (b) show the spatially resolved molecular orbitals at $\bar{\Gamma}$ for each band. In a simplistic view, they are constructed by overlapping different benzene molecular orbitals, which are schematically shown in (c).

The weak interaction observed between the zigzag chain film and the substrate is a favorable playground for a systematic theoretical analysis. As a first approximation, we consider the polymers as free-standing and planar. On this basis, we use DFT calculations to corroborate the weakly dispersive band structure observed experimentally. The calculated electronic structure shown in Figure 2a exhibits convincing qualitative agreement with the experimental data. In particular, the dispersive character of the first four valence bands (VBs) of the zigzag chain (between $-1 \mathrm{eV}$ and $-2.5 \mathrm{eV}$ ) is consistent with that in Figure 1c. The energy mismatch can be attributed to the absence of a substrate in the calculations, as well as to the well-known limitation of DFT to accurately predict HOMO-LUMO gaps. Note that the 
calculated bands span from the $\bar{\Gamma}$ point to the Brillouin zone boundary $(\pi / L)$, which in the experiment appears 12 times replicated until $2 \pi / a^{*}$. For comparison, the calculations are extended to straight PPP chains (Figure 2b) which strongly differ in the electronic structure by exhibiting a highly dispersive single VB in the same energy window. Moreover, the zigzag chain exhibits a greater bandgap than its straight counterpart, confirming the enhanced semiconductive character of the former (see figure S7 in S.I.).

The experimental value of the frontier orbital bandgap of the zigzag chains can be obtained by low-temperature $(4 \mathrm{~K})$ STS. For such measurements we deposit a submonolayer coverage of DMTP molecules on $\mathrm{Ag}(111)$ so that small zigzag island patches are formed on the surface while still allowing access to the bare substrate for tip calibration and treatment (Figure 3 and Fig. S5 in S.I.). Figure 3a shows the dI/dV spectra at the center (red) of a straight arm of a zigzag chain (see figure inset) and the Ag substrate (grey). The VB onset is detected close to $-2.1 \mathrm{~V}$ (coinciding with the ARPES value in Figure 1d) while the conduction band $(\mathrm{CB})$ edge is around $1.6 \mathrm{~V}$ resulting in an overall bandgap of $\sim 3.7 \mathrm{~V}$. Therefore this value is larger than the $3.2 \mathrm{~V}$ reported for PPP chains grown on $\mathrm{Au}(111)^{42}$ and confirms the enhanced semiconducting character of the zigzag chains.

DFT calculations can also shed light onto the effect that the periodically spaced metajunctions have on the overall electronic structure by comparing the spatially resolved molecular orbitals at the $\bar{\Gamma}$ point with the $\pi$ molecular orbitals of benzene (Figure 2c). In the PPP case (Figure 2b), VB and CB are constructed by the overlap of $\Phi_{3}$ and $\Phi_{3}^{*}$ benzene molecular orbitals, respectively. These orbitals present a large electronic weight on the carbon atoms linking the phenyl rings (para-positions), giving rise to highly dispersive valence and conduction bands. Likewise, the less dispersive character of the VB-1 and CB +1 bands can be attributed to the orbital set that exhibits a nodal plane through the para carbon atoms ( $\Phi_{2}$ and $\Phi_{2}^{*}$ orbitals). Contrarily, for the zigzag chains (Figure 2a) the VB and CB are a combination of two degenerate orbitals. ${ }^{7}$ In particular, the VB is made up of $\Phi_{3}$ (straight 


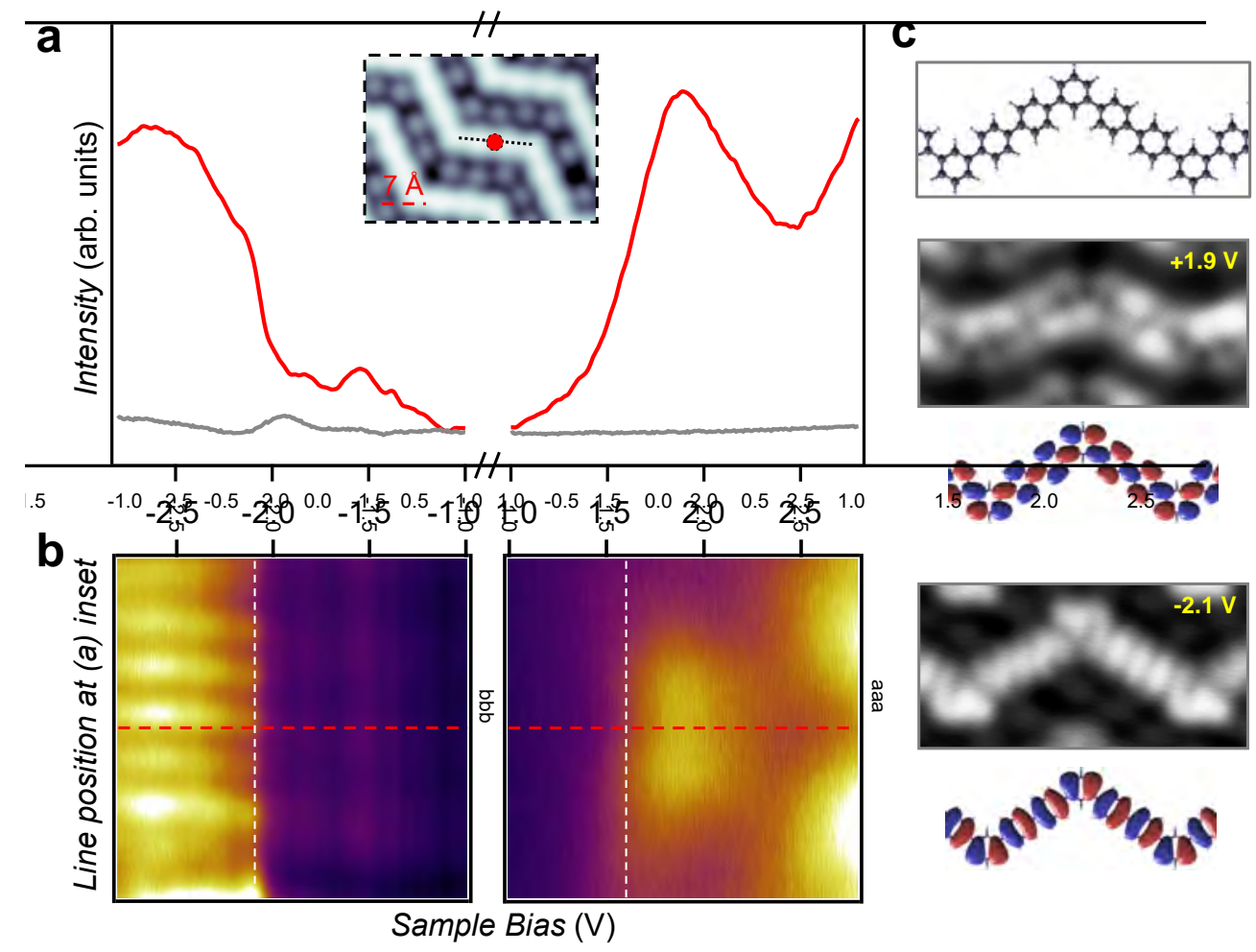

Figure 3: STS experimental determination of the zigzag chain's frontier orbitals on $\mathrm{Ag}(111)$. (a) Constant-height $\mathrm{dI} / \mathrm{dV}$ spectra acquired at the center of a zigzag straight arm (red point in STM image inset) and substrate (grey) (STM imaging parameters: $50 \mathrm{mV}, 100 \mathrm{pA}$; frame: $3.6 \times 2.5 \mathrm{~nm}^{2}$ ). (b) Constant-height $\mathrm{dI} / \mathrm{dV}$ linescan spectra along the zigzag arm (indicated by a dotted line in the STM topography inset) for the same bias range of panel (a). The onsets of the VB and $\mathrm{CB}$ are clearly defined (vertical dashed white lines), yielding a bandgap of $\sim 3.7 \mathrm{eV}$ (STS bias voltage modulation for (a) and (b): $10 \mathrm{mV}_{r m s}$ at $341 \mathrm{~Hz}$. Close-feedback parameters: $-350 \mathrm{mV}, 150 \mathrm{pA}$ and $1200 \mathrm{mV}, 100 \mathrm{pA}$ for the negative and positive resonances regions, respectively). (c) From top to bottom: ball and stick model of the zigzag chain. High-resolution $\mathrm{dI} / \mathrm{dV}$ maps acquired at constant-height with a CO functionalized STM tip at $1.9 \mathrm{~V}$ and $-2.1 \mathrm{~V}$, i.e. close to the $\mathrm{CB}$ and VB onsets (frames: $3.0 \times 1.5 \mathrm{~nm}^{2}$; bias voltage modulation $10 \mathrm{mV}_{r m s}$ at $341 \mathrm{~Hz}$ ). Underneath each map the corresponding DFT gas phase molecular frontier orbitals are shown for comparison. 
sections) and $\Phi_{2}$ (elbows) orbitals, which is mirrored in the CB by $\Phi_{3}^{*}$ (straight sections) and $\Phi_{2}^{*}$ (elbows). This orbital mixing, along with the reduced orbital amplitude at the metapositions and expected phase shifts induced by momentum steering at the elbows, results in a diminished orbital interaction (overlap) that leads to a severe weakening of the electron coupling between adjacent straight segments. Indeed, the flat band character is also exhibited by the VB-1 and $\mathrm{CB}+1$, even though they mostly arise from a single type of benzene molecular orbital coupling $\left(\Phi_{3}\right.$ and $\Phi_{3}^{*}$, respectively). This strong electronic effect governed by the meta-junction is generally referred to as cross-conjugation ${ }^{5,6}$ or destructive quantum interference. $^{8-10,14}$

The reduced electronic coupling between neighboring linear segments causes electron localization, an effect that can be adequately addressed with STS. Figure 3b presents a color plot representing stacked $\mathrm{dI} / \mathrm{dV}$ point spectra measured along a single straight segment (black dashed line in the inset of Fig. 3a). Aside from clearly visualizing an overall bandgap of $3.7 \mathrm{eV}$, we observe confinement in the $\mathrm{CB}$ within such segments where the spatial modulations in the local density of states (LDOS) are consistent with the first two stationary states of a particle in a box. In particular, their amplitudes die away at the edges of the linear segments (elbow positions of the zigzag chains) but the lower state at $1.9 \mathrm{~V}$ features an antinode at the segment's center (the peak in the red spectrum of Fig. 3a), whereas the second state at $2.5 \mathrm{~V}$ oppositely exhibits a node at that position. Such electron confinement effects have also been observed in related structures, as in the case of finite size PPP chains featuring a single elbow (in meta-junction) ${ }^{13}$ or in closed-cycle geometries of honeycombenes. ${ }^{48}$ In essence, we can conclude that meta-junctions act as scattering barriers for the polymer electrons regardless of the overall geometry, i.e. as closed structures ${ }^{48}$ or as edged (non-linear) chains. ${ }^{13}$

Once we have verified that each straight segment of our zigzag chains acts as a confining unit, reminiscent of a 1D array of weakly interacting Quantum Dots (QDs), ${ }^{49}$ it should be possible to tune their electronic properties by modulating the straight segment's 

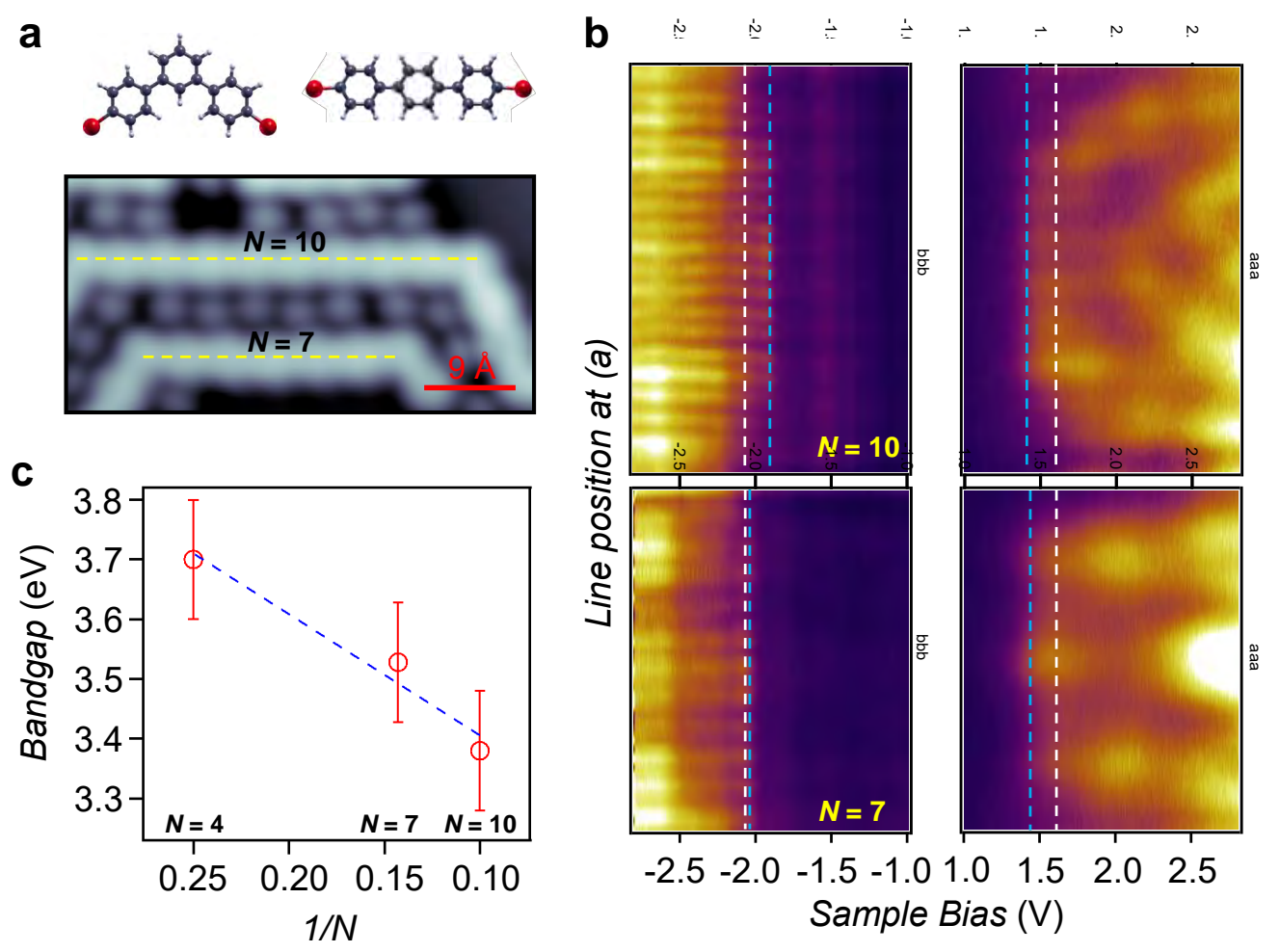

Figure 4: Tuning the electronic confined states through the linear segment's length (QD size). (a) Schematic representation of the co-evaporated molecular precursors (DMTP and DBTP) that generate longer straight segments between the meta-coordinating phenyls. The STM image shows two longer linear segments of 7 and 10 phenyl rings between elbows (Imaging parameters: $50 \mathrm{mV}, 100 \mathrm{pA}$; frame: $4.5 \times 2.3 \mathrm{~nm}^{2}$ ). (b) Constant-height dI/dV line profiles close to the VB and $\mathrm{CB}$ onsets along the two segments following the dashed lines in (a). The intensity modulation of the CB confirms the confinement nature of the metajunction termination. Furthermore, the frontier orbital bandgap (vertical dashed blue lines) is reduced compared to the $N=4$ case, which is indicated by the two vertical dashed white lines. (STS parameters: bias voltage modulation of $10 \mathrm{mV}_{r m s}$ at $341 \mathrm{~Hz}$; close-feedback at $-350 \mathrm{mV}, 150 \mathrm{pA}$ and $1200 \mathrm{mV}, 120 \mathrm{pA}$ for the negative and positive resonances regions, respectively). (c) Experimental bandgap extracted from the STS line profiles revealing a $1 / N$ behavior (compare to Figure S8 in S.I.). 
length. Varying the 1D QD length should affect the energy levels as well as the corresponding frontier orbital bandgap. To do so, we co-evaporated on $\operatorname{Ag}(111)$ linear precursors (DBTP molecules ${ }^{24}$ ) together with the previously used ones to generate the zigzag chains, as shown in Figure 4a. The Ullmann coupling reaction is likewise activated by post-annealing to $470 \mathrm{~K}$, resulting in linear segments of phenyl length configurations of $N=4+3 n$ ( $N$ being the total phenyl number and $n$ the amount of DBTP precursors embedded in the straight segment). Figure $4 \mathrm{~b}$ shows color plots of the $\mathrm{dI} / \mathrm{dV}$ linescan for $N=7$ (bottom) and $N=10$ (top) QDs, which evidence the expected squared wavefunction intensity variations in the CBs for the same energy range as Figure 3b. Most importantly, by comparison to the dashed white lines corresponding to the $N=4$ segment, we observe that the bandgap shrinks as the size of the segment increases (dashed blue lines). A quantitative analysis of the experimentally determined bandgap is shown in Figure 4c, revealing a $1 / N$ behavior in agreement with previous work for similar chains. ${ }^{13}$ Such behavior matches our DFT calculations for planar, free-standing, periodic zigzag chains of different straight segment length, which confirm not only that the bandgap of the cross-conjugated zigzag chains is larger than the one of its linear PPP counterpart, but it is also tunable as $1 / N$ (cf. Figures S7 and S8 in S.I.).

Finally, we should discuss an additional property of this system that may affect the frontier orbital bandgap: the relative twisting of the phenyl rings (non-planar chain morphology). Figure $3 \mathrm{c}$ shows high-resolution constant height $\mathrm{dI} / \mathrm{dV}$ maps close to the valence and conduction band onsets, namely at $-2.1 \mathrm{eV}$ and $1.9 \mathrm{eV}$, respectively. Both maps replicate well the molecular orbital simulations of Figure 2a, which are calculated for planar structures and are depicted below for direct comparison. While the slight discrepancies in nodal positions are attributed to the $\mathrm{CO}$ probe functionalization, ${ }^{50}$ the intensity variations are in turn ascribed to the twisting of the phenyl rings. ${ }^{42}$ The phenyl twisting is confirmed from constant height bond resolution imaging with a $\mathrm{CO}$ functionalized tip, ${ }^{51}$ on which we find subtle intensity modulations on the external parts of the phenyl rings (see Fig. S9 of S.I.) indicative of such 


\section{Conclusions}

We have been able to synthesize and macroscopically align a saturated film of cross-conjugated oligophenylene zigzag chains on a vicinal $\operatorname{Ag}(111)$ surface. We find that these atomically precise chains remain sufficiently decoupled from each other and from the substrate to probe their elusive band structure with ARPES, revealing weakly dispersing one-dimensional electronic bands along the chain direction. DFT band structure calculations and EPWE photoemission intensity simulations satisfactorily reproduce our experimental findings. By means of STS, we find that the zigzag chain has a larger frontier orbital bandgap than its straight counterpart (PPP) and observe electronic confinement in each straight segment of the zigzag chains, reminiscent of 1D arrays of weakly interacting QDs. Such states can be tuned by changing the length of the straight segments, affecting the frontier orbital bandgap, which follows a $1 / N$ dependency. Indeed, our molecular orbital simulations confirm that the periodically spaced meta-junctions at the elbows of the zigzag chain are the main structural feature responsible for the reduction of electronic coupling between adjacent linear segments. These findings corroborate the important effects that the conductive path topology of a molecular 
wire has on its frontier orbitals, which are responsible for defining its chemical, optical and electronic properties. Recent advances in transfer techniques ensure that on-surface synthesized and well-aligned organic nanostructures can be collectively transferred onto insulating substrates maintaining their relative arrangement, ${ }^{52-54}$ which opens the path to further study the transport and optical properties of these cross-conjugated oligophenylene zigzag chains.

\section{Methods}

A silver crystal surface curved around the (645) direction was used as tunable vicinal substrate for chain formation and alignment. ${ }^{29}$ This curved sample exhibits (111) terraces of variable size (position dependent on its curvature) separated by monoatomic steps oriented along the [11-2] direction. The steps are of fully-kinked type, in which out-protruding atoms have no side neighbors (Figure S1 in S.I). Notably, periodic roughening of such step-edges bears negligible energy cost, and hence can readily accommodate to the zigzag structure of the chains. ${ }^{29}$ The saturated zigzag film that presents best order was observed at the vicinal plane $\sim 3.6^{\circ}$ from the (111) region, corresponding to an average terrace size of $3.8 \mathrm{~nm}$. The ARPES data shown in Figure 1 and Figures S1, S3, S4 and S6 in S.I. corresponds to this position of the substrate. The sample was cleaned by repeated cycles of $\mathrm{Ar}^{+}$sputtering at energies of $1.0 \mathrm{keV}$, followed by annealing at $700 \mathrm{~K}$. This produced clean and well-ordered surface step arrays as verified by the splitting of the LEED spots along the surface curvature.

The meta-junctioned haloaromatic compound, DMTP, was sublimated from a Knudsen cell at $360 \mathrm{~K}$ at a low flux (1 ML/hour) while the sample was held at $470 \mathrm{~K} .{ }^{22,48,55}$ Covalently bonded zigzag chains appeared separated by Br adatoms, suggesting some influence from the latter in steering chain growth and alignment. The step flexibility promoted the chain formation while keeping other irregular structures or hyperbenzene macrocycles to a 
minimum. ${ }^{41}$ The structures remained densely packed up to high temperatures $(\sim 600 \mathrm{~K})$, beyond which halogen desorption starts, accompanied by chain misalignment. ${ }^{18,56}$

ARPES measurements were performed with a lab-based experimental setup using a display-type hemispherical electron analyzer (SPECS Phoibos 150, energy/angle resolution of $\left.40 \mathrm{meV} / 0.1^{\circ}\right)$ combined with a monochromatized Helium I $(h \nu=21.2 \mathrm{eV})$ source. Measurements were acquired with the sample at $150 \mathrm{~K}$ by moving the polar angle, which is set to be parallel to the average step direction.

The STM measurements on the curved $\mathrm{Ag}(111)$ crystal were carried out at $100 \mathrm{~K}$ using a variable temperature Omicron STM with a Nanonis SPM control system. The bias voltages given in the manuscript refer to a grounded tip. STM data were acquired in constant current mode and were processed with the WSxM software. ${ }^{57}$

LT-STM/STS measurements were performed with a commercial Scienta-Omicron low temperature system, operating in ultrahigh vacuum (UHV) at $4.3 \mathrm{~K}$. For the measurement, the bias voltage was applied to the tip while the sample was electronically grounded. The STM tip was prepared ex-situ by clipping a Pt/Ir wire $(0.25 \mathrm{~mm})$ and sharpened $i n$-situ by repeatedly indenting the tip a few nanometers (1 to $4 \mathrm{~nm}$ ) into the Ag surface while applying bias voltages from $2 \mathrm{~V}$ to $4 \mathrm{~V}$ between tip and sample. In order to perform bond-resolved STM imaging, the tip apex was terminated with a CO molecule, directly picked up from the surface, by positioning the sharp metal tip on top of it and applying a $500 \mathrm{~ms}$ bias pulse at $-2 \mathrm{~V}$. The imaging was performed by measuring at constant height while applying a bias voltage to the tip within the range of $2.0 \mathrm{mV}$ and $3.5 \mathrm{mV}$. For spectroscopic point spectra and conductance maps, the $\mathrm{dI} / \mathrm{dV}$ signals were measured by a digital lock-in amplifier (Nanonis). STM images were analyzed by using the WSxM software. ${ }^{57}$

Ab-initio calculations were carried out using density functional theory (DFT), as imple- 
mented in the SIESTA code. ${ }^{58}$ The optB88-vdW functional, ${ }^{59}$ which accounts for non-local corrections, was adopted for the exchange and correlation potential. For each organic nanostructure, we considered a supercell consisting of a chain infinite along the $x$ axis, with vacuum gaps of $15 \AA$ in $y$ and $z$ directions in order to avoid interactions between chains in adjacent cells. A Monkhorst-Pack k-point grid with 20x1x1 k-points was used for the Brillouin zone sampling and the mesh cut-off for real space integrations was set to 300 Ry. We employed a double- $\xi$ plus polarization (DZP) basis set, and a mesh-cutoff of 300 Ry for the real-space integrations. All structures were fully relaxed until residual forces were less than $0.01 \mathrm{eV} / \AA$.

The Electron-Plane-Wave-Expansion (EPWE) method, which was recently used to describe the electronic properties of graphene nanostructures, ${ }^{47}$ is employed to simulate ARPES data. Within the EPWE approach, the photoemission intensity for a given binding energy and photoelectron wave vector is obtained from Fermi's golden rule applied to the in-plane wave function (an initial state) and a normalized plane wave (a final state) for the parallel component of the photoelectron wave function, as detailed in Ref. [47]. In this semiempirical method, zigzag chains are considered free-standing and planar, which implies that the simulated bands are substrate independent and free of $\mathrm{Br}$ interactions.

\section{Acknowledgement}

I.P-Z thanks Dr. Jens Brede for fruitful discussion. Z.M.A. thanks Prof. F. J. García de Abajo for providing the EPWE code. We acknowledge the financial support from the Spanish Ministry of Economy, Industry and Competitiveness (MINECO, Grant No. MAT2016-78293C6), from the Basque Government (Grant No. IT-621-13), from the regional Government of Aragon (RASMIA project), from the European Regional Development Fund (ERDF) under the program Interreg V-A España-Francia-Andorra (Contract No. EFA 194/16 TNSI), 
from the German Science Foundation (DFG) through the CRC 1083 and Grant No. GO 1812/2 and funding from the European Research Council (ERC) under the European Union's Horizon 2020 research and innovation programme (grant agreement no. 635919).

\section{Supporting Information Available}

Supporting Information Available: Containing additional information and 10 figures. This material is available free of charge via the Internet at http://pubs.acs.org.

\section{Author Information}

\section{Corresponding Author}

*E-mail: (I.P-Z) ipiquerozulaica@gmail.com and (J.L-C) jorge.lobo@csic.es

\section{Author Contributions}

I.P-Z., J.L-C. and C.K.K. conducted the experimental measurements of the saturated film (ARPES, STM and LEED) and corresponding data analysis; L.C., I.P-Z., M.S. and D.G.O. performed LT-STM/STS measurements of the partially covered surface; A.G-L. performed the DFT calculations and Z.M.A. performed EPWE simulations; I.P-Z. and J.L-C. wrote the manuscript. All authors contributed to the revision and final discussion of the manuscript; I.P-Z., J.L-C., J.E.O. and J.M.G. conceived this project.

\section{Associated Content}

The authors declare that they have no competing financial interests.

\section{References}

(1) Günes, S.; Neugebauer, H.; Sariciftci, N. S. Conjugated Polymer-Based Organic Solar Cells. Chem. Rev. 2007, 10\%, 1324-1338. 
(2) Li, G.; Chang, W.-H.; Yang, Y. Low-Bandgap Conjugated Polymers Enabling SolutionProcessable Tandem Solar Cells. Nat. Rev. Mater. 2017, 2, 17043.

(3) Masai, H.; Terao, J. Stimuli-Responsive Functionalized Insulated Conjugated Polymers. Polym. J. 2017, 49, 805-814.

(4) Guiglion, P.; Zwijnenburg, M. A. Contrasting the Optical Properties of the Different Isomers of Oligophenylene. Phys. Chem. Chem. Phys. 2015, 17, 17854-17863.

(5) Hong, S. Y.; Kim, D. Y.; Kim, C. Y.; Hoffmann, R. Origin of the Broken Conjugation in m-Phenylene Linked Conjugated Polymers. Macromolecules 2001, 34, 6474-6481.

(6) van der Veen, M.; Rispens, M.; Jonkman, H.; Hummelen, J. Molecules with Linear $\pi$ Conjugated Pathways between All Substituents: Omniconjugation. Adv. Funct. Mater. $2004,14,215-223$.

(7) Kocherzhenko, A. A.; Grozema, F. C.; Siebbeles, L. D. A. Single Molecule Charge Transport: From a Quantum Mechanical to a Classical Description. Phys. Chem. Chem. Phys. 2011, 13, 2096-2110.

(8) Manrique, D. Z.; Huang, C.; Baghernejad, M.; Zhao, X.; Al-Owaedi, O. A.; Sadeghi, H.; Kaliginedi, V.; Hong, W.; Gulcur, M.; Wandlowski, T.; Bryce, M. R.; Lambert, C. J. A Quantum Circuit Rule for Interference Effects in Single-Molecule Electrical Junctions. Nat. Commun. 2015, 6, 6389.

(9) Guédon, C. M.; Valkenier, H.; Markussen, T.; Thygesen, K. S.; Hummelen, J. C.; van der Molen, S. J. Observation of Quantum Interference in Molecular Charge Transport. Nat. Nanotechnol. 2012, 7, 305-309.

(10) Markussen, T.; Stadler, R.; Thygesen, K. S. The Relation between Structure and Quantum Interference in Single Molecule Junctions. Nano Lett. 2010, 10, 4260-4265. 
(11) Limacher, P. A.; Lüthi, H. P. Cross-Conjugation. Wiley Interdiscip. Rev. Comput. Mol. Sci. 2011, 1, 477-486.

(12) Thompson, A. L.; Ahn, T.-S.; Thomas, K. R. J.; Thayumanavan, S.; Martínez, T. J.; Bardeen, C. J. Using Meta Conjugation To Enhance Charge Separation versus Charge Recombination in Phenylacetylene Donor-Bridge-Acceptor Complexes. J. Am. Chem. Soc. 2005, 127, 16348-16349.

(13) Wang, S.; Wang, W.; Lin, N. Resolving Band-Structure Evolution and Defect-Induced States of Single Conjugated Oligomers by Scanning Tunneling Microscopy and TightBinding Calculations. Phys. Rev. Lett. 2011, 106, 206803.

(14) Tada, T.; Yoshizawa, K. Molecular Design of Electron Transport with Orbital Rule: Toward Conductance-Decay Free Molecular Junctions. Phys. Chem. Chem. Phys. 2015, 17, 32099-32110.

(15) Terao, J.; Wadahama, A.; Matono, A.; Tada, T.; Watanabe, S.; Seki, S.; Fujihara, T.; Tsuji, Y. Design Principle for Increasing Charge Mobility of $\pi$-Conjugated Polymers Using Regularly Localized Molecular Orbitals. Nat. Commun. 2013, 4, 1691.

(16) Cai, J.; Ruffieux, P.; Jaafar, R.; Bieri, M.; Braun, T.; Blankenburg, S.; Muoth, M.; Seitsonen, A. P.; Saleh, M.; Feng, X.; Müllen, K.; Fasel, R. Atomically Precise BottomUp Fabrication of Graphene Nanoribbons. Nature 2010, 466, 470-473.

(17) Talirz, L.; Ruffieux, P.; Fasel, R. On-Surface Synthesis of Atomically Precise Graphene Nanoribbons. Adv. Mater. 2016, 28, 6222-6231.

(18) de Oteyza, D. G.; Garcia-Lekue, A.; Vilas-Varela, M.; Merino-Díez, N.; CarbonellSanromà, E.; Corso, M.; Vasseur, G.; Rogero, C.; Guitián, E.; Pascual, J. I.; Ortega, J. E.; Wakayama, Y.; Peña, D. Substrate-Independent Growth of Atomically Precise Chiral Graphene Nanoribbons. ACS Nano 2016, 10, 9000-9008. 
(19) Corso, M.; Carbonell-Sanromà, E.; de Oteyza, D. G. In On-Surface Synthesis II; de Oteyza, D. G., Rogero, C., Eds.; Springer International Publishing: Cham, 2018; pp $113-152$.

(20) Gröning, O.; Wang, S.; Yao, X.; Pignedoli, C. A.; Borin Barin, G.; Daniels, C.; Cupo, A.; Meunier, V.; Feng, X.; Narita, A.; Müllen, K.; Ruffieux, P.; Fasel, R. Engineering of Robust Topological Quantum Phases in Graphene Nanoribbons. Nature 2018, 560, 209-213.

(21) Rizzo, D. J.; Veber, G.; Cao, T.; Bronner, C.; Chen, T.; Zhao, F.; Rodriguez, H.; Louie, S. G.; Crommie, M. F.; Fischer, F. R. Topological Band Engineering of Graphene Nanoribbons. Nature 2018, 560, 204-208.

(22) Fan, Q.; Wang, C.; Han, Y.; Zhu, J.; Hieringer, W.; Kuttner, J.; Hilt, G.; Gottfried, J. M. Surface-Assisted Organic Synthesis of Hyperbenzene Nanotroughs. Angew. Chem. Int. Ed. 2013, 52, 4668-4672.

(23) Vasseur, G.; Fagot-Revurat, Y.; Sicot, M.; Kierren, B.; Moreau, L.; Malterre, D.; Cardenas, L.; Galeotti, G.; Lipton-Duffin, J.; Rosei, F.; Di Giovannantonio, M.; Contini, G.; Le Fèvre, P.; Bertran, F.; Liang, L.; Meunier, V.; Perepichka, D. F. Quasi One-Dimensional Band Dispersion and Surface Metallization in Long-Range Ordered Polymeric Wires. Nat. Commun. 2016, \%, 10235.

(24) Basagni, A.; Vasseur, G.; Pignedoli, C. A.; Vilas-Varela, M.; Peña, D.; Nicolas, L.; Vitali, L.; Lobo-Checa, J.; de Oteyza, D. G.; Sedona, F.; Casarin, M.; Ortega, J. E.; Sambi, M. Tunable Band Alignment with Unperturbed Carrier Mobility of On-Surface Synthesized Organic Semiconducting Wires. ACS Nano 2016, 10, 2644-2651.

(25) Abadía, M.; Ilyn, M.; Piquero-Zulaica, I.; Gargiani, P.; Rogero, C.; Ortega, J. E.; Brede, J. Polymerization of Well-Aligned Organic Nanowires on a Ferromagnetic RareEarth Surface Alloy. ACS Nano 2017, 11, 12392-12401. 
(26) Ortega, J. E.; Mugarza, A.; Repain, V.; Rousset, S.; Pérez-Dieste, V.; Mascaraque, A. One-Dimensional versus Two-Dimensional Surface States on Stepped Au(111). Phys. Rev. B 2002, 65, 165413.

(27) Mugarza, A.; Schiller, F.; Kuntze, J.; Cordón, J.; Ruiz-Osés, M.; Ortega, J. E. Modelling Nanostructures with Vicinal Surfaces. J. Phys.: Condens. Matter 2006, 18, S27.

(28) Corso, M.; Schiller, F.; Fernández, L.; Cordón, J.; Ortega, J. E. Electronic States in Faceted Au(111) Studied with Curved Crystal Surfaces. J. Phys.: Condens. Matter 2009, 21, 353001 .

(29) Ortega, J. E.; Vasseur, G.; Piquero-Zulaica, I.; Matencio, S.; Valbuena, M. A.; Rault, J. E.; Schiller, F.; Corso, M.; Mugarza, A.; Lobo-Checa, J. Structure and Electronic States of Vicinal $\operatorname{Ag}(111)$ Surfaces with Densely Kinked Steps. New J. Phys. 2018, 20, 073010 .

(30) Ruffieux, P.; Cai, J.; Plumb, N. C.; Patthey, L.; Prezzi, D.; Ferretti, A.; Molinari, E.; Feng, X.; Müllen, K.; Pignedoli, C. A.; Fasel, R. Electronic Structure of Atomically Precise Graphene Nanoribbons. ACS Nano 2012, 6, 6930-6935.

(31) Senkovskiy, B. V.; Usachov, D. Y.; Fedorov, A. V.; Haberer, D.; Ehlen, N.; Fischer, F. R.; Grüneis, A. Finding the Hidden Valence Band of $\mathrm{N}=7$ Armchair Graphene Nanoribbons with Angle-Resolved Photoemission Spectroscopy. 2D Mater. 2018, 5, 035007 .

(32) Cai, L.; Yu, X.; Liu, M.; Sun, Q.; Bao, M.; Zha, Z.; Pan, J.; Ma, H.; Ju, H.; Hu, S.; Xu, L.; Zou, J.; Yuan, C.; Jacob, T.; Björk, J.; Zhu, J.; Qiu, X.; Xu, W. Direct Formation of C-C Double-Bonded Structural Motifs by On-Surface Dehalogenative Homocoupling of gem -Dibromomethyl Molecules. ACS Nano 2018, 12, 7959-7966.

(33) Merino-Díez, N.; Lobo-Checa, J.; Nita, P.; Garcia-Lekue, A.; Basagni, A.; Vasseur, G.; Tiso, F.; Sedona, F.; Das, P. K.; Fujii, J.; Vobornik, I.; Sambi, M.; Pascual, J. I.; 
Ortega, J. E.; de Oteyza, D. G. Switching from Reactant to Substrate Engineering in the Selective Synthesis of Graphene Nanoribbons. J. Phys. Chem. Lett. 2018, 9, $2510-2517$.

(34) Bieri, M.; Nguyen, M.-T.; Gröning, O.; Cai, J.; Treier, M.; Aït-Mansour, K.; Ruffieux, P.; Pignedoli, C. A.; Passerone, D.; Kastler, M.; Müllen, K.; Fasel, R. TwoDimensional Polymer Formation on Surfaces: Insight into the Roles of Precursor Mobility and Reactivity. J. Am. Chem. Soc. 2010, 132, 16669-16676.

(35) Judd, C. J.; Haddow, S. L.; Champness, N. R.; Saywell, A. Ullmann Coupling Reactions on $\operatorname{Ag}(111)$ and $\operatorname{Ag}(110)$; Substrate Influence on the Formation of Covalently Coupled Products and Intermediate Metal-Organic Structures. Sci. Rep. 2017, 7, 14541.

(36) Wang, W.; Shi, X.; Wang, S.; Van Hove, M. A.; Lin, N. Single-Molecule Resolution of an Organometallic Intermediate in a Surface-Supported Ullmann Coupling Reaction. J. Am. Chem. Soc. 2011, 133, 13264-13267.

(37) Di Giovannantonio, M.; El Garah, M.; Lipton-Duffin, J.; Meunier, V.; Cardenas, L.; Fagot Revurat, Y.; Cossaro, A.; Verdini, A.; Perepichka, D. F.; Rosei, F.; Contini, G. Insight into Organometallic Intermediate and Its Evolution to Covalent Bonding in Surface-Confined Ullmann Polymerization. ACS Nano 2013, 7, 8190-8198.

(38) Fan, Q.; Wang, C.; Han, Y.; Zhu, J.; Kuttner, J.; Hilt, G.; Gottfried, J. M. SurfaceAssisted Formation, Assembly, and Dynamics of Planar Organometallic Macrocycles and Zigzag Shaped Polymer Chains with C-Cu-C Bonds. ACS Nano 2014, 8, 709-718.

(39) Koch, M.; Gille, M.; Viertel, A.; Hecht, S.; Grill, L. Substrate-Controlled Linking of Molecular Building Blocks: $\mathrm{Au}(111)$ vs. Cu(111). Surf. Sci. 2014, 627, 70-74.

(40) Chen, M.; Shang, J.; Wang, Y.; Wu, K.; Kuttner, J.; Hilt, G.; Hieringer, W.; Gottfried, J. M. On-Surface Synthesis and Characterization of Honeycombene Oligophenylene Macrocycles. ACS Nano 2017, 11, 134-143. 
(41) Fan, Q.; Wang, T.; Dai, J.; Kuttner, J.; Hilt, G.; Gottfried, J. M.; Zhu, J. On-Surface Pseudo-High-Dilution Synthesis of Macrocycles: Principle and Mechanism. ACS Nano 2017, 11, 5070-5079.

(42) Merino-Díez, N.; Garcia-Lekue, A.; Carbonell-Sanromà, E.; Li, J.; Corso, M.; Colazzo, L.; Sedona, F.; Sánchez-Portal, D.; Pascual, J. I.; de Oteyza, D. G. WidthDependent Band Gap in Armchair Graphene Nanoribbons Reveals Fermi Level Pinning on $\mathrm{Au}(111)$. ACS Nano 2017, 11, 11661-11668.

(43) Fan, Q.; Liu, L.; Dai, J.; Wang, T.; Ju, H.; Zhao, J.; Kuttner, J.; Hilt, G.; Gottfried, J. M.; Zhu, J. Surface Adatom Mediated Structural Transformation in Bromoarene Monolayers: Precursor Phases in Surface Ullmann Reaction. ACS Nano 2018, 12, $2267-2274$.

(44) Offenbacher, H.; Lüftner, D.; Ules, T.; Reinisch, E. M.; Koller, G.; Puschnig, P.; Ramsey, M. G. Orbital Tomography: Molecular Band Maps, Momentum Maps and the Imaging of Real Space Orbitals of Adsorbed Molecules. J. Electron Spectrosc. Relat. Phenom. 2015, 204, 92-101.

(45) Koller, G.; Berkebile, S.; Oehzelt, M.; Puschnig, P.; Ambrosch-Draxl, C.; Netzer, F. P.; Ramsey, M. G. Intra- and Intermolecular Band Dispersion in an Organic Crystal. Science 2007, 317, 351-355.

(46) Mugarza, A.; Mascaraque, A.; Pérez-Dieste, V.; Repain, V.; Rousset, S.; García de Abajo, F. J.; Ortega, J. E. Electron Confinement in Surface States on a Stepped Gold Surface Revealed by Angle-Resolved Photoemission. Phys. Rev. Lett. 2001, 87, 107601.

(47) Abd El-Fattah, Z. M.; Kher-Elden, M. A.; Piquero-Zulaica, I.; García de Abajo, F. J.; Ortega, J. E. Graphene: Free Electron Scattering Within an Inverted Honeycomb Lattice. arXiv:1808.06034 
(48) Fan, Q.; Dai, J.; Wang, T.; Kuttner, J.; Hilt, G.; Gottfried, J. M.; Zhu, J. Confined Synthesis of Organometallic Chains and Macrocycles by Cu-O Surface Templating. ACS Nano 2016, 10, 3747-3754.

(49) Piquero-Zulaica, I.; Lobo-Checa, J.; Sadeghi, A.; Abd El-Fattah, Z. M.; Mitsui, C.; Okamoto, T.; Pawlak, R.; Meier, T.; Arnau, A.; Ortega, J. E.; Takeya, J.; Goedecker, S.; Meyer, E.; Kawai, S. Precise Engineering of Quantum Dot Array Coupling Through Their Barrier Widths. Nat. Commun. 2017, 8, 787.

(50) Gross, L.; Moll, N.; Mohn, F.; Curioni, A.; Meyer, G.; Hanke, F.; Persson, M. HighResolution Molecular Orbital Imaging Using a p-Wave STM Tip. Phys. Rev. Lett. 2011, $107,086101$.

(51) Hieulle, J.; Carbonell-Sanromà, E.; Vilas-Varela, M.; Garcia-Lekue, A.; Guitián, E.; Peña, D.; Pascual, J. I. On-Surface Route for Producing Planar Nanographenes with Azulene Moieties. Nano Lett. 2018, 18, 418-423.

(52) Ohtomo, M.; Sekine, Y.; Hibino, H.; Yamamoto, H. Graphene Nanoribbon Field-Effect Transistors Fabricated by Etchant-Free Transfer from Au(788). Appl. Phys. Lett. 2018, 112, 021602.

(53) Llinas, J. P. et. al. Short-Channel Field-Effect Transistors with 9-Atom and 13-Atom Wide Graphene Nanoribbons. Nat. Commun. 2017, 8, 633.

(54) Moreno, C.; Vilas-Varela, M.; Kretz, B.; Garcia-Lekue, A.; Costache, M. V.; Paradinas, M.; Panighel, M.; Ceballos, G.; Valenzuela, S. O.; Peña, D.; Mugarza, A. BottomUp Synthesis of Multifunctional Nanoporous Graphene. Science 2018, 360, 199-203.

(55) Fan, Q.; Gottfried, J. M.; Zhu, J. Surface-Catalyzed C-C Covalent Coupling Strategies toward the Synthesis of Low-Dimensional Carbon-Based Nanostructures. Acc. Chem. Res. 2015, 48, 2484-2494. 
(56) Goddard, P.; Schwaha, K.; Lambert, R. Adsorption-Desorption Properties and Surface Structural Chemistry of Bromine on Clean and Sodium-Dosed Ag(111). Surf. Sci. 1978, $71,351-363$.

(57) Horcas, I.; Fernández, R.; Gómez-Rodríguez, J. M.; Colchero, J.; Gómez-Herrero, J.; Baro, A. M. WSXM: A Software for Scanning Probe Microscopy and a Tool for Nanotechnology. Rev. Sci. Instrum. 2007, 78, 013705.

(58) Soler, J. M.; Artacho, E.; Gale, J. D.; García, A.; Junquera, J.; Ordejón, P.; SánchezPortal, D. The SIESTA Method for Ab Initio Order- $N$ Materials Simulation. J. Phys.: Condens. Matter 2002, 14, 2745-2779.

(59) Klimeš, J.; Bowler, D. R.; Michaelides, A. Chemical Accuracy for the Van Der Waals Density Functional. J. Phys.: Condens. Matter 2010, 22, 022201. 


\section{Graphical TOC Entry}

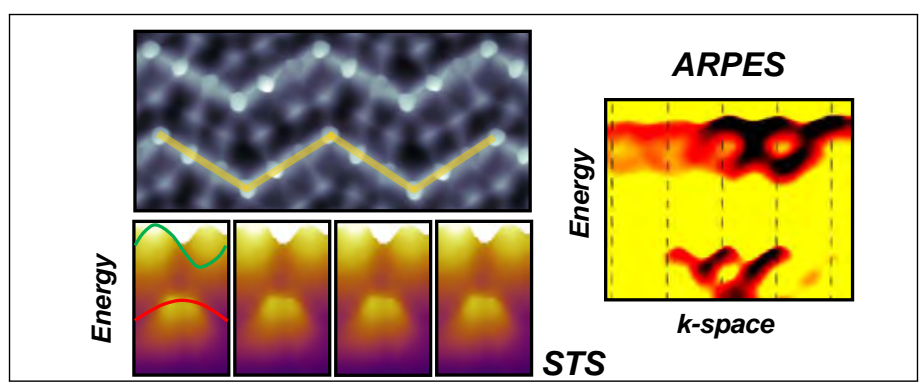

